\title{
The new age of cytopathology: applications of maldi imaging and super-resolution microscopy
}

\section{Editorial}

The art of establishing pathological diagnosis based on the microscopic analysis of clinical samples is challenging. Cytopathology is characterized by the morphological, anatomical and molecular analysis of cells and tissues for the accurate diagnosis of clinical features of patients suffering from various pathophysiological conditions ranging from infectious diseases to cancer. The diagnostic work-flow of cytopathology involves active collaboration efforts between clinicians and pathologists. The impact of cytopthology is enormous in the diagnosis and prognosis of cancer. The diagnosis of tumor biopsy is performed to confirm the underlying neoplastic or nonneoplastic nature of tumor. However for the sake of accurate diagnosis of cancer obtaining information both at spatial and molecular levels is vital. Matrix-assisted laser desorption/ionization (MALDI)-based mass spectrometry imaging (IMS) and super-resolution microscopy (SRM) have emerged as cutting edge techniques in deciphering the molecular information with spatial resolution in clinical samples.

The advent of Matrix-assisted laser desorption/ionization (MALDI)-based mass spectrometry imaging uncover new opportunities for the clinicians and pathologists to extract molecular and spatial information from tissues. MALDI time-of-flight (TOF) imaging mass spectrometry (IMS) is an exciting technology which cemented its place in clinical diagnosis especially in oncology. It harnesses the advantages of mass spectrometry in detecting biomolecules with complementary spatial resolution in clinical tissue samples. ${ }^{1}$ However the challenge remains is this field is the low sensitivity which is characterized by the poor ionization efficiency of numerous bio-molecules that are present in tissue slices. ${ }^{2}$ A giant leap of this technology came in the form of MALDI-2 which improves the sensitivity of MALDI imaging by up to two orders of magnitude thereby facilitating the detection of bio-molecules of different origins such as protein, lipids, carbohydrates and proteins. ${ }^{2}$

The application of this technique is valuable in the identification of tumor heterogeneity, tumor origin in metastatic tissues, grading of cancer stages and prognostic markers where conventional cytopathology techniques struggle due to the inability in obtaining detailed spatial molecular information from clinical samples. ${ }^{3}$ Since tissues in pathological conditions harbor the all important information of genetic and proteomic alteration thereby mapping the molecular alteration in the tissues will provide an array of information to pin point the underlying pathological condition. For this reason MALDI IMS applicability is not restricted to cancer diagnosis only but to various diseases such as cardiovascular, ${ }^{4}$ gastrointestinal, ${ }^{5}$ renal diseases. ${ }^{6}$

In this post genomic era the concept of personalized medicine is not a far-fetching idea but emerged as a realistic approach to treat individual patients. However a major challenge in the personalized medicine for cancer is the tumor heterogeneity. The reason behind it boils down to the fact that tumors grow and develops in its own unique manner in one individual which may differ from other individuals. This remarkable heterogeneity requires unique therapeutic approach that may vary from one individual to another. To decipher the long
Volume 2 Issue 2 - 2017

\author{
Sajib Chakraborty, Hossain Uddin Shekhar \\ Department of Biochemistry and Molecular Biology, University \\ of Dhaka, Bangladesh
}

Correspondence: Department of Biochemistry and Molecular Biology, University of Dhaka, Dhaka-1000, Bangladesh, Email hossainshekhar@du.ac.bd

Received: February 18, 2017 | Published: February 20, 2017

standing complexity of tumor heterogeneity MALDI IMS with its high spatial resolution and molecular phenotyping approach can potentially be used to resolve the phenotypic variation that exists in a tumor entity in one individual. ${ }^{7}$ The spatial resolution of MALDI IMS gives this technique a cutting edge over DNA- and RNA-based molecular methods since the spatial information is completely lost during the preparative phase of nucleic acids.

MALDI imaging with its capacity to resolve molecular information with spatial resolution cannot replace the requirement of microscopic analysis. In order to obtain essential information about the morphology and anatomical structures the application of microscopic analysis is immense in the field of pathology. However traditional fluorescence microscopy technique is restricted by the resolution limit set by the diffraction of light that greatly hinders the information gathering capacity resulting in low resolution images of the clinical samples. Therefore conventional microscopy cannot be used to analyze the detailed molecular organization of proteins in tissue samples. However in the past years we have witnessed the emergence of Superresolution microscopy (SRM) in resolving the molecular structures beyond the diffraction-limited resolution of traditional microscopes. ${ }^{8}$ Formalin fixed and/or paraffin embedded tissues obtained during surgery are underutilized but can potentially provide diagnostic insights for therapy and prognosis. The use of optical microscopy of these tissues is limited due to the diffraction-limited resolution. In recent years stimulated emission depletion (STED) based superresolution microscopy technique-free of diffraction-limited resolution has been used to visualize nano-scale protein localization in paraffinembedded human rectal cancer tissue. ${ }^{9}$ Another evolving field where SRM may play crucial role in deciphering the complexity is structural organization of neurons in the brain. The link among protein localization, neuron architecture and neuronal communication has already been emerged as complex circuit to govern brain activity. The prospect of SRM in gathering the detailed information of molecular organization and neuronal structure is immense and could proved to be vital in designing therapeutic approach for neurodegenerative disorder. ${ }^{10}$

Altogether the prospects of cytology and pathology in the modern era have grown immensely owing to the discovery of ground-breaking techniques such as MALDI imaging and SRM. The Journal-Advances 
in Cytology \& Pathology is committed to publish high impact research papers with the aim to encourage scientists world-wide by recognizing their contribution in this exciting field and uploading the scientific break-through and insights.

\section{Acknowledgements}

None.

\section{Conflict of interest}

The author declares no conflict of interest.

\section{References}

1. Caprioli RM, Farmer TB, Gile J. Molecular imaging of biological samples: localization of peptides and proteins using MALDI-TOF MS. Anal Chem. 1997;6(9):4751-4760.

2. Soltwisch J, Kettling H, Vens Cappell S, et al. Mass spectrometry imaging with laser-induced postionization. Science. 2015;348(6231):211215.

3. Kriegsmann J, Kriegsmann M, Casadonte R. MALDI TOF imaging mass spectrometry in clinical pathology: A valuable tool for cancer diagnostics. International journal of oncology. 2014;46(3):893-906.
4. Thomas A, Lenglet S, Chaurand P, et al. Mass spectrometry for the evaluation of cardiovascular diseases based on proteomics and lipidomics. Thromb Haemost. 2011;106(1):20-33.

5. Seeley EH, Washington MK, Caprioli RM, et al. Proteomic patterns of colonic mucosal tissues delineate Crohn's colitis and ulcerative colitis. Proteomics Clin Appl. 2013;7(7-8):541-549.

6. Lalowski M, Magni F, Mainini V. Imaging mass spectrometry: a new tool for kidney disease investigations. Nephrol Dial Transplant. 2013;28(7):1648-1656.

7. Schone C, Hofler H, Walch A. MALDI imaging mass spectrometry in cancer research: combining proteomic profiling and histological evaluation. Clin Biochem. 2013;46(6):539-545.

8. Perkel JM. Superresolution microscopy. Science. 2016;352(6287):850 852.

9. Ilgen P, Stoldt S, Conradi LC, et al. STED super-resolution microscopy of clinical paraffin-embedded human rectal cancer Tissue. Plos one. 2014;9(7):e101563.

10. Maglione M, Sigrist SJ. Seeing the forest tree by tree: super-resolution light microscopy meets the neurosciences. Nature Neuroscience. 2013;16(7):790-797. 